\title{
Photoelectron spectroscopy as a non-invasive method to monitor SASE-FEL spectra
}

\author{
M. Wellhöfer, ${ }^{a}$ J.T. Hoeft, ${ }^{a}$ M. Martins, ${ }^{a *}$ W. Wurth, ${ }^{a}$ M. Braune, ${ }^{b}$ J. Viefhaus, ${ }^{b c}$ \\ K. Tiedtke ${ }^{c}$ and M. Richter ${ }^{d}$ \\ a Institute of Experimental Physics, Luruper Chaussee, \\ 149, 22761 Hamburg, Germany, \\ ${ }^{b}$ Fritz-Haber-Institut der Max-Planck-Gesellschaft, Abt. Molekülphysik, \\ Faradayweg 4-6, 14195 Berlin, Germany \\ ${ }^{c}$ HASLYAB, DESY, Notkestr. 85, 22761 Hamburg, Germany \\ ${ }^{d}$ Physikalisch-Technische Bundesanstalt, \\ Abbestr. 2-12, 10587 Berlin, Germany \\ E-mail: michael.martins@desy.de
}

ABSTRACT: Since the summer of 2005, the vacuum ultra-violet Free-electron LASer in Hamburg (FLASH) has operated as a user facility at the Deutsches Elektronen-Synchrotron (DESY), delivering ultra-short laser pulses of tens of femtosecond duration with a high peak brilliance of up to $10^{28}$ photons $/\left(\mathrm{s} \mathrm{mm}^{2} \mathrm{mrad}^{2} 0.1 \%\right.$ bandwidth). Due to the statistics of the Self-Amplified Spontaneous Emission (SASE) process, each photon pulse differs from the previous one in the number of modes per pulse, the wavelength ( $0.5 \%$ fluctuations) and the intensity, making experiments more complicated. Thus, for certain experiments the detailed knowledge of the beam properties on a shot-to-shot basis is mandatory. In this paper we describe an online method to gain spectral information about the individual Free-Electron Laser (FEL) pulses that is based on rare-gas photoionization and photoelectron spectroscopy.

KEYWORDS: Instrumentation for FEL; Vacuum-based detectors; Gaseous detectors; Interaction of radiation with matter.

${ }^{*}$ Corresponding author. 


\section{Contents}

1. Introduction 1

2. Experimental setup 2

3. Results and discussion 4

4. Conclusion and outlook

\section{Introduction}

At third-generation storage rings for the generation of synchrotron radiation, the photon flux slowly decreases with time, but does not fluctuate from pulse to pulse. The pulse duration is typically several tens of picoseconds and therefore much longer than the time-scale of electronic relaxation in matter. On the other hand, the new soft x-ray Free-electron LASer in Hamburg (FLASH) at DESY [1] can generate VUV-laser pulses that are some tens of femtoseconds in pulse duration and with a peak brilliance of up to $10^{28}$ photons $/\left(\mathrm{s} \mathrm{mm}^{2} \mathrm{mrad}^{2} 0.1 \%\right.$ bandwidth). This is accomplished by taking advantage of the so-called Self Amplified Spontaneous Emission (SASE) process [2, 3]. The short and intense laser pulses can be used for element specific studies, e.g., the dynamics of chemical reactions, of materials, and of atomic systems in general. However, due to the statistical nature of the SASE process, FLASH does not show a distinct, stable spectrum, the mean photon energy fluctuates by about $0.5 \%$, the number of modes per pulse fluctuate and the intensity also fluctuates from shot to shot. This statistical behavior has been studied theoretically [ [ $]$ ] and needs to be taken into account when free-electron laser (FEL) experiments at FLASH are planned. It also leads to specific requirements for the experimental setup and the data acquisition system. Since complex and totally new experiments are planned to be performed at FLASH one needs to understand the artifacts that can arise while doing measurements with such a new and unique facility. We therefore began by studying well-known atomic systems, i.e. the photoionization of rare gases, by electron spectroscopy. Our photoelectron spectra measured within the linear regime of photonmatter interaction represent a convolution of the respective well-known rare gas electron energy distribution with the photon energy spectrum of the incident FLASH beam. Thus, the latter may be derived from our data and so represents a new technique for online spectrometry. However, at high levels of laser intensity and target gas density, the high number of created electron-ion pairs may influence the spectra by plasma effects like electron-ion coulomb interaction as well as electronelectron repulsion. We therefore measured and controlled pulse energy, peak photon exposure, target gas density, and photon energy during our experiments. For the latter, the monochromator beamline PG at FLASH has a special option to use zeroth-order and first-order light simultaneously. The gas phase experiments were carried out utilizing zeroth-order light, whereas in first order the individual spectrum of the respective FEL pulse was monitored. Furthermore, a built-in gas monitor detector [5] (GMD) allowed the acquisition of the intensity levels of the individual photon pulses with an uncertainty of $10 \%$. 


\section{Experimental setup}

A schematic view of the experimental setup is depicted in figure 1. The master clock triggers the whole FEL as well as all attached measurement devices such as cameras and fast digitizer cards. The master clock also generates a unique bunch train number for each bunch train which is then distributed to all devices. All measuring devices are connected via ethernet to the central data acquisition system called DAQ [6]. ${ }^{1}$ The accumulated data are read out by the computer and send to the DAQ together with the unique bunch train number. The bunch trains have a repetition rate of $2 \mathrm{~Hz}$ or $5 \mathrm{~Hz}$, respectively. Each bunch train consisted of up to 30 pulses, all separated by $1 \mu \mathrm{s}$.

Photoelectron spectra of rare gases have been acquired at the zeroth order branch PG0 with two different setups. The first setup refers to angle-resolved photoelectron emission spectroscopy. It is equipped with electron Time-of-Flight (eTOF) spectrometers ${ }^{2}$ and allows the fast detection of the emitted electrons even for a complete bunch train. The interaction region is actively magnetically screened from the residual earth magnetic field by three Helmholtz coil pairs. The second setup consists of a vacuum chamber with a SCIENTA SES 200 hemispherical electron spectrometer. ${ }^{3}$ Here, the magnetic screening of the interaction region was realized by an in-situ permalloy shielding. In both systems, the background gas pressure in the respective recipient was varied from $3 \times 10^{-7}$ mbar up to $3 \times 10^{-5}$ mbar. It was dosed via a capillary of $500 \mu \mathrm{m}$ inner diameter placed $5-8 \mathrm{~mm}$ away from the interaction region. It resulted in a gas jet with an estimated local pressure being one to two orders of magnitude higher than the residual gas pressure measured.

Photoelectron spectra of $\mathrm{He}, \mathrm{Ne}$, and Xe were acquired using the eTOF setup. In addition, we investigated the influence of the residual pressure on the shape of the photoelectron spectra with the SCIENTA setup. This has been carried out for the rare gases $\mathrm{He}, \mathrm{Ne}, \mathrm{Ar}, \mathrm{Kr}$, and Xe. Since the readout speed of the ICCD and CCD cameras was limited to $10 \mathrm{~Hz}$ we used single pulse mode throughout our experiments. The FLASH beam is fully horizontally polarized. The angular distribution of the photoelectrons is described by the $\beta$ parameter according to [7]:

$$
\frac{d \sigma}{d \Omega}=\frac{\sigma}{4 \pi}\left(1+\beta P_{2}(\cos \theta)\right)
$$

with $\theta$ the angle of emission with respect to the polarization axis of the incident photon beam, $d \Omega$ the solid angle of acceptance, $\sigma$ the cross section, and $P_{2}$ the Legendre polynomial of second order. $\beta$ varies between $\beta=0.6$ and $\beta=2.0$ for the relevant $s$ - or $p$-shell single photon absorption process at $38 \mathrm{eV}$ incident photon energy (see table 1). Therefore, our detectors were constantly held at zeroth degree with respect to the polarization axis in order to gain maximum possible photoelectron yield. Taking into account all figures of merit (e.g. solid angle of acceptance, number

\footnotetext{
${ }^{1}$ The measurement systems normally consist of a Standard PC with a 24 bit Input/Output register from National Instruments PCI-6503 and the device itself, e.g., firewire CCD camera, ICCD camera, or digitizer card. Recently, this has been changed to a more time accurate method, where we use IP Timer cards on each computer to encode the bunch train number into the data output of the attached device.

${ }^{2}$ For the readout of the eTOF, we used an Acqiris Digitizer Card DP120 with 8 bit per data point and 2GS/s sampling rate connected to a standard Windows PC. At a later stage we also used the Acqiris digitizer system DP282 with 10 bit resolution and $8 \mathrm{GS} / \mathrm{s}$ sampling rate on a LINUX system. The later system was equipped with an IP timer card for more accurate timing.

${ }^{3}$ The MCP signals of the electron analyzer were detected via a CCD firewire camera BASLER 104f.
} 


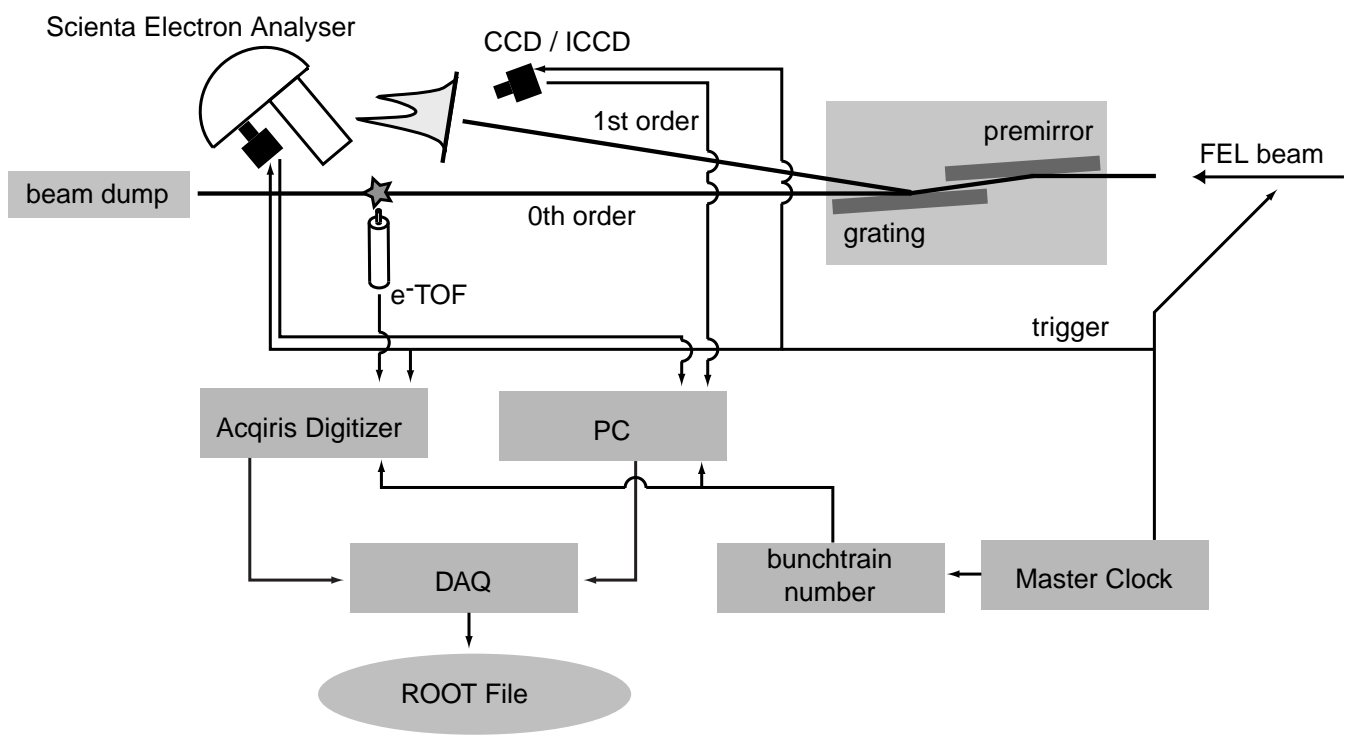

Figure 1. Schematic view of the experimental setup for the simultaneous measurement of first order VUVFEL spectra and photoelectron spectra

Table 1. Cross section $\sigma$ (taken from ref. [9]), $\beta$ value, and ionization threshold $I_{1}$ (taken from ref. [7] and references therein) for the different rare gases at $38 \mathrm{eV}$ incident photon energy. The last column denotes the ratio of the mean free path of the helium atoms to the mean free path of the respective other rare gases [10] in the pressure range of $10^{-5}$ mbar.

\begin{tabular}{|lccc|c|}
\hline & $\sigma(\mathrm{Mb})$ & $\beta$ & $\mathrm{I}_{1}(\mathrm{eV})$ & $k_{\text {helium }} / k_{x}$ \\
\hline $\mathrm{He}(1 \mathrm{~s})$ & 3.5 & 2.0 & 24.6 & 1.00 \\
$\mathrm{Ne}(2 \mathrm{p})$ & 8.79 & 0.6 & $21.6,21.7$ & 0.68 \\
$\mathrm{Ar}(3 \mathrm{p})$ & 4.69 & 1.6 & $15.8,15.9$ & 0.34 \\
$\mathrm{Kr}(4 \mathrm{p})$ & 5.85 & 1.8 & $14.0,14.7$ & 0.27 \\
$\mathrm{Xe}(5 \mathrm{p})$ & 2.47 & 2.0 & $12.1,13.4$ & 0.20 \\
\hline
\end{tabular}

of atoms within the interaction region, ionization cross section of the individual gas, photon beam intensity) an estimated number of a few thousand electrons reached the detector per photon pulse. Therefore, the MCP of the eTOF had to operate in a charge accumulation mode. An amplifier converted the charge pulses into voltage pulses which were then measured as a function of time with a fast analog-to-digital converter with high bandwidth. All acquired data were immediately sent to the DAQ where they were written to ROOT files [8] for further analysis.

In the first-order branch PG2, the dispersed FEL radiation could be monitored utilizing a Ce:YAG screen [11] with camera system. ${ }^{4}$ The spectrum of each individual pulse was acquired. The GMD detector for measuring the intensity was located in front of the mirror grating unit (not shown in figure 1).

\footnotetext{
${ }^{4}$ ANDOR iStar ICCD or Firewire CCD camera BASLER 304f, respectively
} 


\section{Results and discussion}

We first measured the FLASH photon energy spectrum near $93 \mathrm{eV}$ on a shot-to-shot basis and correlated our measurement with the intensity of the individual photon pulses. We sorted the spectra according to their intensity and built an average photon energy distribution. Normally, one would not expect any systematic dependence of the mean photon energy with the intensity fluctuations at a specific point of machine operation. The photon pulses are generated by a short electron pulse moving through a long section of undulators. As a result of the interaction of the generated light with the electrons, the electron pulse gets microbunched more and more which finally results in the characteristic SASE FEL radiation. Thus, specific regions of FEL operation (e.g. linear vs. saturation) correspond, for a given undulator length, to certain electron beam parameters [2]. In figure 2 , the mean photon energy is depicted as a function of photon pulse energy (number of photons) for a specific point in the linear (open circles) and the saturation regime (grey filled rectangles) of FLASH. The two regimes of operation can be distinguished not only by the magnitude of the mean pulse energy but, moreover, also by their pulse energy distribution (PED). In figure 3(a) the PED is depicted in the case of the linear regime. Within this regime, the PED follows a gamma distribution [12]. From the fit to the gamma distribution (solid line), the mean fluctuation can be derived and thus the mean number of modes per pulse which is the inverse square of the deviation. In our case the deviation in pulse energy is $61 \%$. In the linear regime the deviation of pulse energy increases steadily with increasing mean pulse energy as the degree of microbunching of the electron pulse evolves exponentially along the undulator. When entering the saturation regime the fluctuations of pulse energy drops at a specific mean pulse energy (see figure 3(b) as an example for the deviation of pulse energy in the saturation regime); the degree of microbunching of the electron pulse is close to one now. The quick drop of the deviation in radiation energy is a feature of the ultra-short pulse duration [13]. Going deeper into saturation the overall beam is stabilized; photon pulse lengthening also begins to occur because a significant part of the long tail of the electron pulse starts lasing. This again increases the spectral width of the FEL photon pulses (see also figure $\$$ ).

In the linear regime, the mean photon energy quickly drops to a saturation value with increasing number of photons (figure 22). The mean photon energy is directly correlated with the kinetic energy of the electrons lasing. In the current operation mode of FLASH the electron pulse has a complicated shape and reveals a systematic kinetic energy distribution (within 1\%) with the sharply rising edge of the pulse having higher kinetic energy than the slowly falling tail. As a result the mean photon energy shifts with increasing photon beam intensity to lower values. In the saturation regime, then, no further shift of the photon energy is observable within the measurement uncertainties as the whole electron pulse now lases and therefore the photon beam is stabilized.

A shift in mean photon energy was also observed at a mean photon energy of $38 \mathrm{eV}$ as depicted in figure 4 . The graph shows a colored map of the mean photon energy distribution for the regime of deep saturation.

These two examples clearly illustrate that detailed knowledge about the spectral shape of each individual pulse is extremely important for the interpretation of acquired FEL data as a function of the pulse energy.

We next measured the pressure dependence of the kinetic energy distribution of the outer shell photoelectrons for the rare gases helium to xenon at a mean photon energy of $38 \mathrm{eV}$. The 


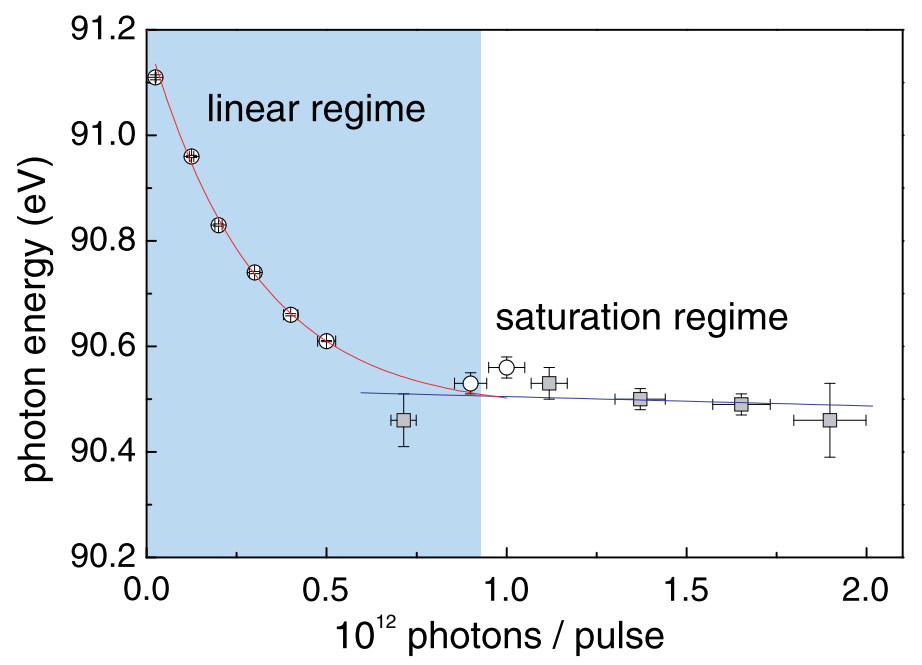

Figure 2. Mean photon energy vs. beam intensity: The mean energy has been acquired by Gaussian fits to the data. The open circles show the mean energy of the run in the linear regime of operation. The red solid line is an exponential decay fit to the open circle data points. The grey rectangles show the mean energy of the run at saturation as a function of the number of photons. The blue line represents a linear fit to the corresponding data points of the run at saturation. The blue shaded area is the pulse intensity range of the linear regime.

lowest pressure value applied was $3.5 \times 10^{-7} \mathrm{mbar}$ and the highest $2.7 \times 10^{-5} \mathrm{mbar}$, limited by the differential pumping stage at the time. As the experimental setup in zeroth order, we chose the SCIENTA system. In first order, we acquired the SASE spectra simultaneously. FLASH was operating in the single bunch mode at a repetition rate of $2 \mathrm{~Hz}$. The experiment was carried out with a vertically unfocused beam of $3 \mathrm{~mm}$ height and a horizontal focus of $100 \mu \mathrm{m}$, therefore, the photon exposure in the interaction region was rather low $\left(<10^{10}\right.$ photons $\left./ \mathrm{cm}^{2}\right)$ and non-linear effects [1417] did not play any role. For different gas pressure values, we evaluated the influence of electronion and electron-electron coulomb interaction on the spectra. We therefore measured the photolines of the rare gases at low and high pressure and sorted the line spectra according to the incoming photon beam intensity. Due to the high energy resolution of the electron spectrometer $(50 \mathrm{eV}$ pass energy for the SCIENTA, better than $0.3 \%$ for the eTOF), the width of the photoelectron lines were dominated by the spectral bandpass of the laser pulses. We then calculated the effective binding energy of the outer shell electrons, i.e. the mean photon energy minus the mean kinetic energy. To study the coulomb interaction effects, which should depend on the number of electron-ion pairs created, we subtracted the calculated effective binding energies of the low pressure measurements from that of the high pressure measurements. This was performed for different number of photons per pulse and different rare gases to cover a wide range of created electron-ion pairs. The number of electron-ion pairs $N_{\text {pairs }}$ created depends on the gas-target density $n_{\mathrm{gas}}$, which is proportional to the measured pressure $p$, the number of photons $N_{\text {photon }}$ and the cross section $\sigma_{\mathrm{gas}}$ of the rare gas 


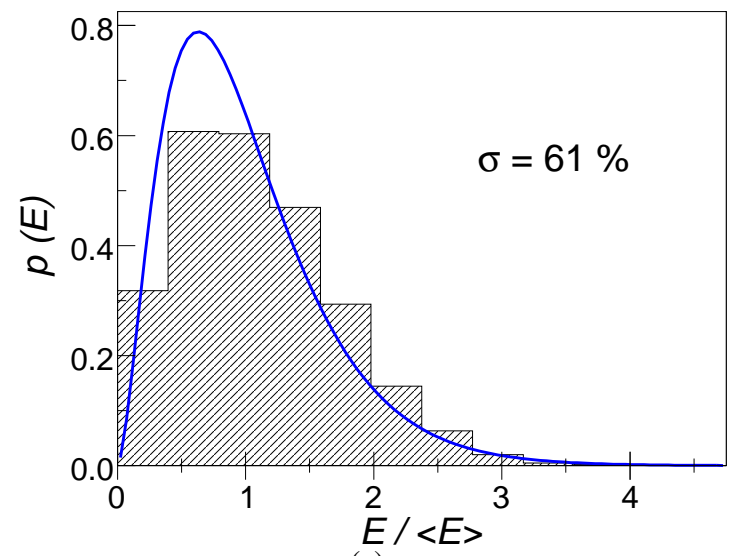

(a)

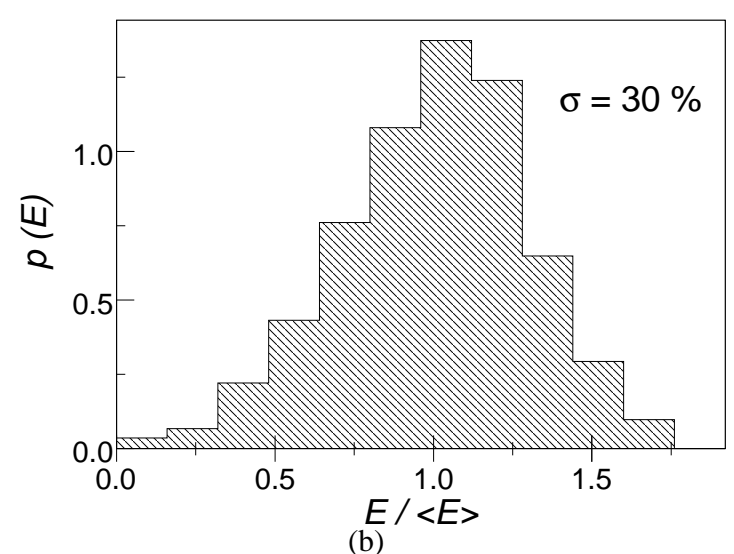

Figure 3. Pulse energy distribution: The $x$-axis is normalized to the average radiation energy in the pulse. Pulse energy distribution for the linear regime (a): The solid curve is a fit with the gamma distribution [12]. From the fit the average number of modes in the radiation pulse $M=2.7$, which is the inverse square of $\sigma$, is derived. (b) pulse energy distribution for the saturation regime

used and was calculated according to:

$$
N_{\text {pairs }}=N_{\text {photon }} \cdot n_{\text {gas }} \cdot \sigma_{\text {gas }} \cdot l .
$$

$l$ denotes the interaction length. $n_{\text {gas }}$ is equal to $p / k_{B} T$ for an ideal gas with $k_{B}$ the Boltzmann constant, $T$ the absolute temperature and $p$ the target-gas pressure in the interaction region. The latter was measured with an ionization gauge and corrected with the respective gas type correction factor. Due to the direct injection of the gas into the interaction region via the capillary, the pressure was assumed to be two orders of magnitude higher than the reading of the ionization gauge. The total error in our pressure measurement is assumed to be one order of magnitude. Because the case of an ideal gas does not hold we additionally took the mean free path $k$ [10] as an estimate for the target gas density. Helium with the longest mean free path was considered as the closest to the case of an ideal gas. A shorter mean free path $k$ is related to stronger interaction of the particle and, thus, a higher particle density. Therefore, we corrected the pressure readings with $k_{\text {helium }} / k_{\mathrm{x}}$ (see last column in table 1). 


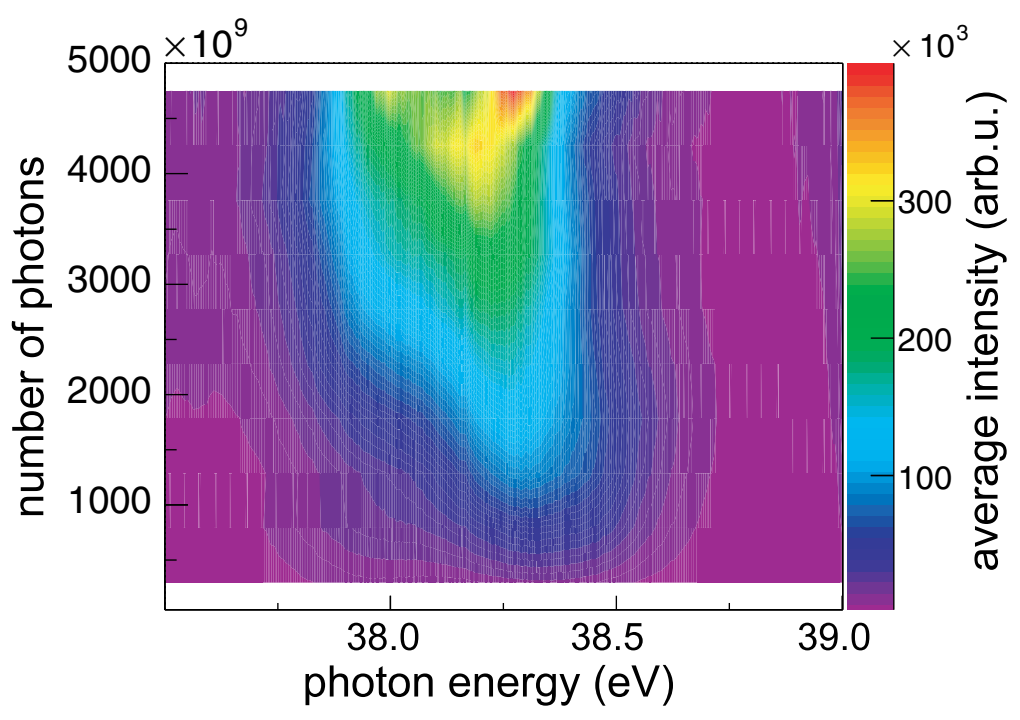

Figure 4. Number of photons vs. photon energy: The intensity ranges from zero (blue violet) to the maximum intensity detected (red). The mean photon energy shifts towards lower energy with higher number of photons per pulse.

In figure 5, the effective binding energy shift for the photoelectrons of $\mathrm{He}, \mathrm{Ne}, \mathrm{Ar}, \mathrm{Kr}$, and $\mathrm{Xe}$ is depicted as a function of electron-ion pairs created. The jitter in average photon energy has been subtracted. The photolines of $\mathrm{He}, \mathrm{Ar}, \mathrm{Kr}$, and Xe linearly shift to lower energies with increasing number of created electron-ion pairs. The Ne line with the highest cross section appears to slightly deviate from the linear behavior above at $4 \times 10^{7}$ created electron-ion pairs. Further measurements are needed to verify that this is really beyond the experimental uncertainties. From the estimation of the local pressure within the interaction region and the uncertainties of our measurement, we can set an upper limit for the onset of kinetic energy shift for the given energy of 15 to $25 \mathrm{eV}$, which occurs at $10^{7}$ electron-ion pairs created. This behavior of kinetic energy shift has also been observed in experiments conducted by Pietzsch et al. [18]. The photoline shift can be explained by the outgoing electron cloud being decelerated by the positive charge of the ions. Due to the very short ionization time, the ions are considered as stationary yielding a stationary electric field wherein the electrons move. Slower electrons were more severely affected by the attractive Coulomb potential of the ions than faster ones. However, in our case the kinetic energy of the photo emitted electrons are of the same order of magnitude for the different rare gases and therefore the effect of Coulomb attraction was also in the same range. In a simple model of a cylindrical capacitor with the ions on the photon beam axis and the electrons moving radially outbound, the energy loss of the electrons due to Coulomb attraction is on the order of $1 \mathrm{eV}$ for $10^{8}$ electron-ion pairs created and increases linearly with increasing number of electron-ion pairs. This is modelled by the blue solid curve in figure 5 .

If the number of generated electron-ion pairs is high, additional pulse broadening occurs. This effect has been studied with the eTOF apparatus. In figure 6 an example of the photon energy distribution of the incident photon pulse and the corresponding eTOF spectrum are depicted. In both spectra, three peaks are clearly visible. The peaks in the photon energy spectrum correspond to three different laser modes present within the single pulse. Due to their statistical origin, these 


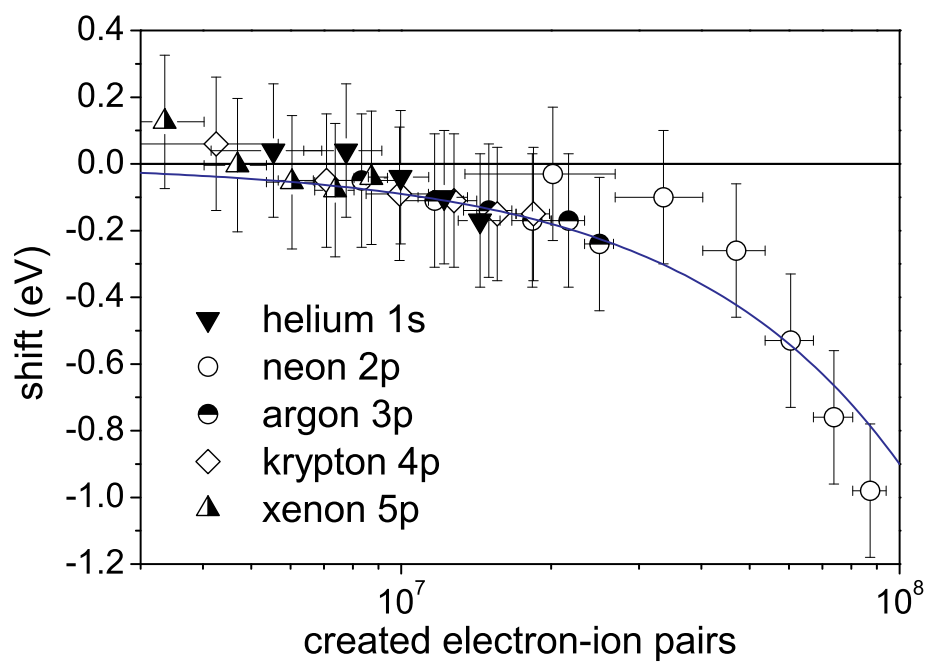

Figure 5. Shift of the kinetic energy of the outgoing s or p electron of the outer shell for different rare gases as a function of number of created electron-ion pairs. The incident photon energy was $38 \mathrm{eV}$. The blue solid curve shows the shift as a guide for the eye.

modes vary from shot to shot in their number, their individual intensity, and their individual photon energy within the bandwidth of the FEL. The bottom figure (b) shows the corresponding electron kinetic energy spectrum. However, due to the repulsive Coulomb force between the electrons, the individual electron peaks broaden and drift apart. Electrons at the head of the cloud with high kinetic energy additionally gain energy as they are repelled from the electrons at the tail of the cloud with lower kinetic energy.

As a result, the mean kinetic energy of the broadened electron cloud is shifted to lower kinetic energy. The broadening depends very much on the number of electrons in the pulse and thus the incident number of photons. In the depicted case, the pulse broadening for the single peaks is about a factor of two with an overall broadening of the entire spectrum of 1.5. Furthermore, the microstructure of the pulses, e.g. maximum to minimum and relative peak height, could not be resolved due to instrumental broadening (time resolution of the digitizer and MCP response function). Roughly $50 \%$ of the acquired spectra show a clear coincidence of the number of peaks; from $15 \%$ of the spectra acquired, the details of the spectral microstructure could be retrieved; these are mainly spectra with single peak structure.

Finally, controlling all figures of merit, e.g. gas pressure, gas type, and photon exposure level, we studied the photoelectron spectra of neon and helium with respect to the question whether it will be possible to retrieve a full spectrum of the incident photon beam by analyzing the shape of the neon $2 p$ or helium 1 s line, respectively. The other rare gases like argon, krypton, and xenon are less suitable due to their more complex electronic structure and their energy splitting of the $p$ photoelectron emission lines on the order of several hundreds of meV. Here, we have chosen the eTOF apparatus since it allowed shot-to-shot measurements within a bunch train of consecutive 


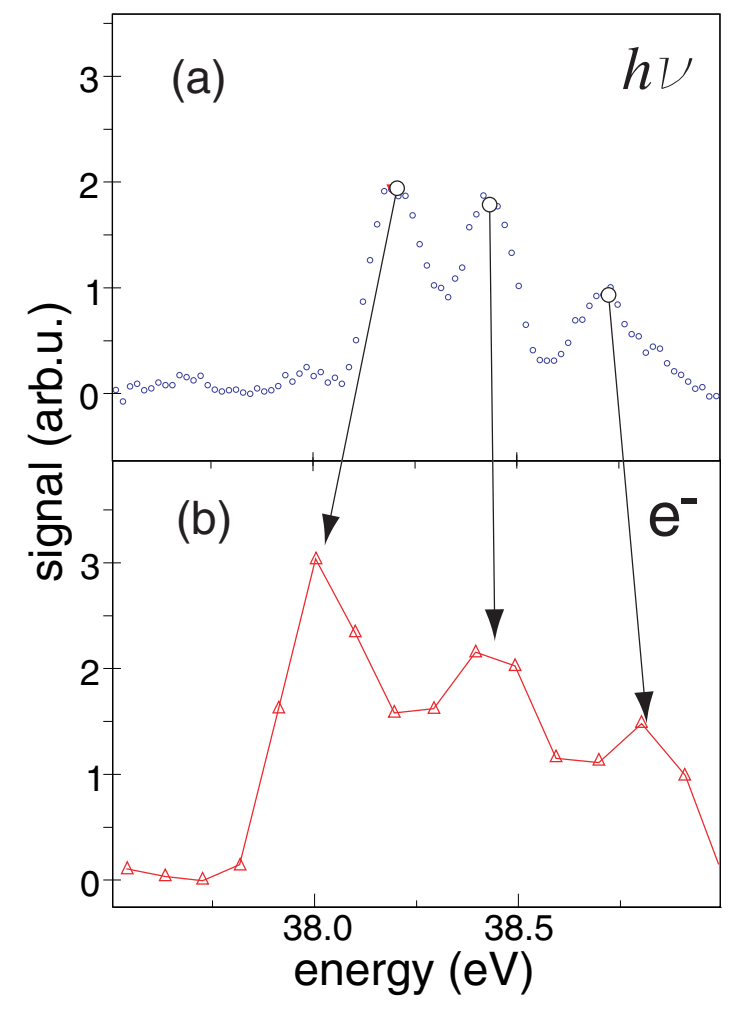

Figure 6. Simultaneously acquired FLASH spectrum of a single shot (a), blue open circles, and corresponding eTOF spectrum (b), red open triangles: For the latter, the binding energy of helium of $24.6 \mathrm{eV}$ has been added to the calculated kinetic energy values to match the photon energy scale.

photon pulses separated by $1 \mu \mathrm{s}$. On the other hand and in contrast should we choose to use the SCIENTA electron analyzer, we would have to operate in single bunch mode with a photon pulse spacing of $500 \mathrm{~ms}$ due to the slow read out rate of the camera system.

However, the eTOF detection method influences the measured kinetic energy distribution of the photoelectrons. Whereas in the SCIENTA electron analyzer the kinetic energy is measured independently from the source point of the electrons, this is not the case for the eTOF spectrometer. Here, the time-of-flight is affected by the path length the electrons have to travel and their kinetic energy. The resolving power, calculated from the kinetic energy $E=1 / 2 \cdot m \cdot x^{2} / t^{2}$ and its derivatives $\partial E / \partial x=\Delta E / \Delta x$ and $\partial E / \partial t=\Delta E / \Delta t$, is then described by

$$
\frac{E}{\Delta E}=\frac{x}{2 \Delta x}+\frac{t}{2 \Delta t}
$$

where $x=309 \mathrm{~mm}$ is the path length from the interaction region to the detector, $\Delta x$ is the source size of the interaction region, $t$ is the time-of-flight and $\Delta t$ is the time resolution of the ADC and the MCP response time. The photon beam was horizontally polarized. The eTOF spectrometer was at $\theta=0^{\circ}$ degree with respect to the polarization axis and had an acceptance length of $3 \mathrm{~mm}$ along the photon beam path. The small horizontal width of $50 \mu \mathrm{m}$ to $100 \mu \mathrm{m}$ of the photon beam allowed a maximum possible resolving power of the eTOF.

Furthermore, the photoelectron flux had to be optimized. On the one hand it had to be reduced 


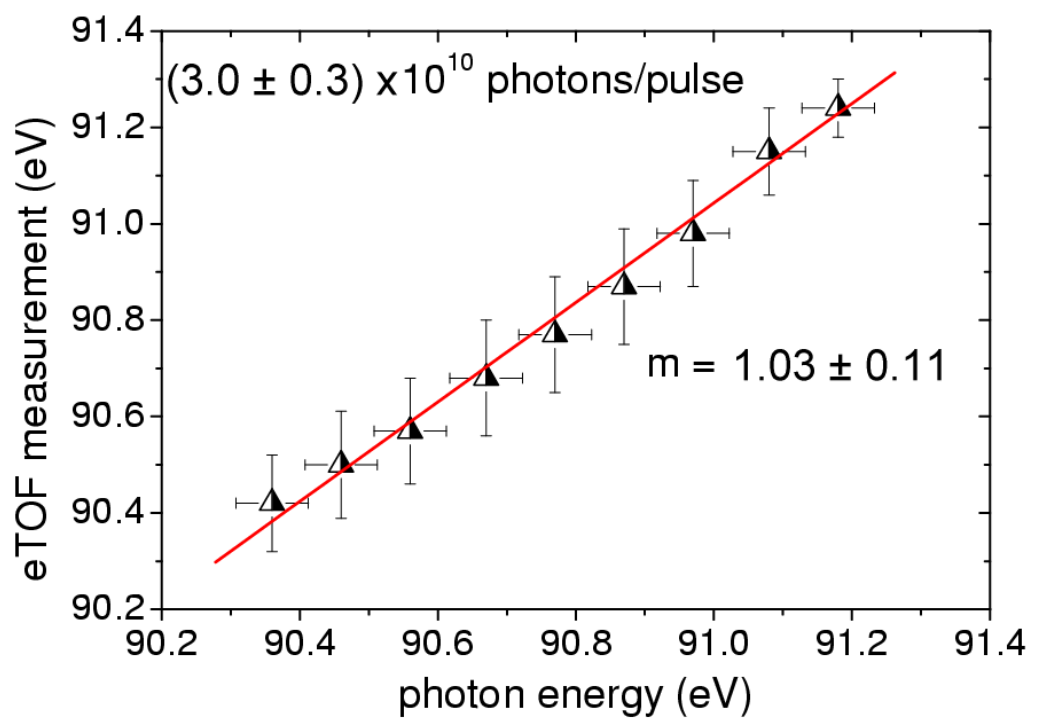

Figure 7. Mean energy value retrieved by eTOF measurement vs. mean photon energy for constant photon intensity: The red solid line is the linear fit to the data points. The slope $m$ is equal to one within the measurement uncertainties. The data points are retrieved from single shot measurements binned within a photon energy interval of $0.105 \mathrm{eV}$ (x-error bar). The $\mathrm{y}$-uncertainties are the statistical errors of the mean kinetic energy distribution for the given photon energy. The number of photons was $(3.0 \pm 0.3) \times 10^{10}$ photons estimated from the theoretical transmission of the beamline [19] and the GMD measurement.

to a level where ion-electron and electron-electron interaction are negligible. On the other hand it had to be sufficiently high to yield enough photoelectrons to be detected to gain a full spectral distribution of the kinetic energy of the photoelectrons within one shot. Therefore, we reduced the transmission of the beamline in zeroth order to about $6 \%$ by inserting baffles in front of the monochromator or choosing monochromator settings for the zeroth order in order to minimize the photon flux. For the given photon exposure levels the gas pressure was chosen in such a way that the number of electrons entering the eTOF detector was about $10^{3}$. This number has proven to be sufficiently high to yield smooth spectra on the one hand and negligible electron-electron repulsion resulting in electron pulse broadening on the other.

In a first step, we measured the kinetic energy distribution of the $2 p$ photoelectron line of neon at a mean incident photon energy of $90 \mathrm{eV}$. The kinetic energy of the emitted electrons was about $68 \mathrm{eV}$. The electrons travel about $30 \mathrm{~mm}$ before they enter the spectrometer. To get a high energy resolution, a retarding potential of $-65 \mathrm{eV}$ was applied within the spectrometer. The time resolution of the fast digitizer was $0.5 \mathrm{~ns}$ which is of the order of the MCP response time of the detector of $0.6 \mathrm{~ns}$. This pushes our resolving power to 300 . However, the retarding field lowered the transmission inside the eTOF spectrometer to critical values. The electric fields may also influence the electron charge distribution within the pulse [18]. The corresponding spectra of the eTOF and the photon pulse did not show an correlation due to the high noise level in the eTOF spectra. Nevertheless, we were able to retrieve the mean photon energy on a shot-to-shot basis. The results for a constant number of photons are depicted in figure 7. As expected the slope $m$ is equal to one within the measurement uncertainties. The data points are retrieved from single shot measurements 


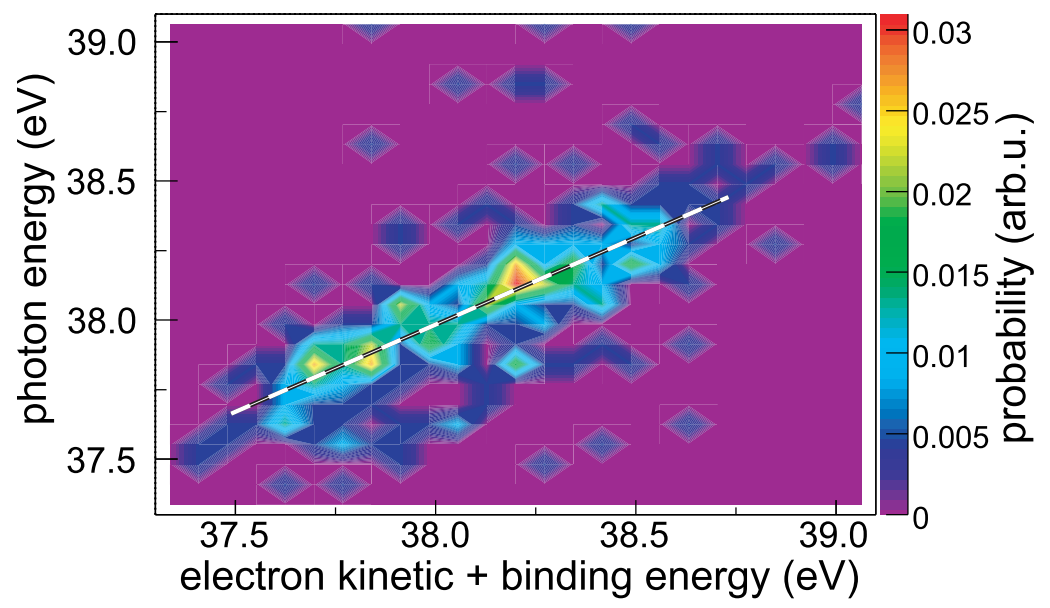

Figure 8. Peak positions of the kinetic energy spectrum of the helium 1s photoelectrons vs. peak positions of the FLASH photon energy: The binding energy of the helium $1 \mathrm{~s}$ line of $24.6 \mathrm{eV}$ has been added. The data were acquired in the single shot mode with a repetition rate of $2 \mathrm{~Hz}$. The black and white dashed line is the linear fit to the data points.

averaged within a photon energy interval of $0.105 \mathrm{eV}$ (x-error bar). The y-uncertainties are statistical errors of the kinetic energy energy distribution for the respective average photon energy. The number of photons was $(3.0 \pm 0.3) \times 10^{10}$ photons estimated from the theoretical transmission of the beamline [19] and the GMD measurements.

Helium has proven to be the better choice since the He 1s line is very narrow compared to the incident bandwidth of the FLASH pulses. Advantageously, it does not have an energy level splitting in contrast to the neon $2 p$ level. Measurements of the kinetic energy distribution of the 1 s photoelectron at a photon energy of $38 \mathrm{eV}$ have been carried out. This time we did not apply a retarding field, which would lower the transmission of the eTOF. The time resolution of $0.5 \mathrm{~ns}$ of the digitizer gave us an energy resolution of roughly $100 \mathrm{meV}$ for the given eTOF parameter. As mentioned earlier, the number of electrons play a crucial role in the formation of the signal detected. We investigated both, photon and photoelectron spectra recorded at PG2 and PG0 simultaneously. Utilizing a peak finding algorithm [20, 21], we found a match of number of peaks and position of the peaks in the photoelectron and photon spectra. The result is displayed in the histogram in figure 8. The colored map shows the probability distribution of the coincidence of photon and photoelectron peak positions. Red denotes to a high probability of coincidence, blue to a low. Normally, one would expect a linear dependence of slope one of the photon and photoelectron peak positions. However, we observe a slope less than one $(0.64 \pm 0.15)$, which can be explained by electron-electron repulsion as discussed above. Often, the electron distribution within one pulse does not consist of one Gaussian pulse, but of a complex superposition of several pulses as a result of the complex photon energy distribution. As discussed, due to electron-electron repulsion the photoelectron spectrum is stretched with respect to the photon spectrum (see figure 6). Thus, the peaks in the photoelectron spectra are further apart than their corresponding peaks in the photon spectra. This explains our deviation from our expected slope of one in figure 8 . 


\section{Conclusion and outlook}

In conclusion, we studied Coulomb effects on photoelectron spectroscopy of rare gases at high photon beam irradiance levels and high target gas pressure. The mean photon energy of the FLASH photon pulses could be retrieved from photoelectron spectra on a shot-to-shot basis. Furthermore, the photoelectron spectra showed coulomb effects at high irradiance levels. This Coulomb effect increases with increasing incident FLASH power. Detailed spectral information about the FLASH pulses could be retrieved for helium at low irradiance levels and low target gas density. In the multi-peak spectra the electron-electron repulsion is strong, thus, stretching the measured spectrum. Retarding electric fields influence the shape of the kinetic energy spectrum of a single shot negatively. For an application of photoelectron spectroscopy as a non-invasive method to monitor SASE FEL photon spectra, one has to keep in mind that the source size limits the resolving power. For the detections system, hemispherical electron analyzers are less suitable due to their electrostatic lenses distorting the microstructure of the electron pulse and their low read-out speed. The eTOF spectrometer has the advantages of the fast read-out speed and its low distortion of the kinetic energy spectrum. Since the number of photons within one pulse from a SASE FEL fluctuates by one order of magnitude, this technique is not reliable as the linearity of the retrieved signal strongly depends on the number of photons. To overcome this disadvantage one could think of a sequence of eTOF spectrometer operating at different gas target pressures. Still the problem of resolving the microstructure of the pulses originating from SASE FEL remain. However, if there is only one mode per shot as in the case of a seeded SASE FEL, it is possible to retrieve the photon energy within one shot. This technique could be used as a diagnostic tool for the X-FEL where reflective gratings are not applicable to determine the photon energy.

\section{Acknowledgments}

This work was supported by the German Ministry of Education and Research through grant 05 KS4GU1/9 and the Helmholtz Joint Research Center Physics with Coherent Radiation Sources. We gratefully acknowledge the FLASH team at HASYLAB/DESY for running the accelerator and for their help in preparing the experiments.

\section{References}

[1] V. Ayvazyan et al., First operation of a free-electron laser generating $G W$ power radiation at $32 \mathrm{~nm}$ wavelength, Eur. Phys. J. D 37 (2006) 297.

[2] E. Saldin, E. Schneidmiller and M. Yurkov, Statistical properties of the radiation from VUV FEL at DESY operating at $30 \mathrm{~nm}$ wavelength in the femtosecond regime, Nucl. Instrum. Meth. A 562 (2006) 472 .

[3] J. Rossbach, A VUV free electron laser at the TESLA test facility at DESY, Nucl. Instrum. Meth. A 375 (1996) 269.

[4] E. Saldin, E. Schneidmiller and M. Yurkov, Simulation studies of $6 \mathrm{~nm}$ free electron laser at the TESLA test facility starting from noise, Nucl. Instrum. Meth. A 393 (1997) 157.

[5] M. Richter et al., Measurement of gigawatt radiation pulses from a vacuum and extreme ultraviolet free-electron laser, Appl. Phys. Lett. 83 (2003) 2970. 
[6] A. Agababyan et al., Data Acquisition System for a VUV-FEL Linac, Conf. Proc. of PCaPAC Hayama, Japan (2005).

[7] U. Becker and D.A. Shirley, VUV and soft x-ray photoionization, Plenum Press, New York (1996).

[8] ROOT, Root - an object oriented data analysis framework, http://root.cern.ch (2006).

[9] J. Samson and W. Stolte, Precision measurements of the total photoionization cross-sections of He, $\mathrm{Ne}, \mathrm{Ar}, \mathrm{Kr}$, and Xe, J. Electron. Spectrosc. Relat. Phenom. 123 (2002) 265.

[10] A. Roth, Vacuum sealing techniques, Springer (1994).

[11] T. Martin and A. Koch, Recent developments in X-ray imaging with micrometer spatial resolution, J. Sync. Rad. 13 (2006) 180.

[12] E. Saldin, E-A-Schneidmiller and M. Yurkov, The physics of free electron lasers, Springer Heidelberg, New York (1999).

[13] V. Ayvazyan et al., Study of the statistical properties of the radiation from a VUV SASE FEL operating in the femtosecond regime, Nucl. Instrum. Meth. A 507 (2003) 368.

[14] Y. Nabekawa, H. Hasegawa, E. Takahasi and K. Midorikawa, Production of doubly charged helium ions by two-photon absorption of an intense Sub-10-fs soft X-ray pulse at $42 \mathrm{eV}$ photon energy, Phys. Rev. Lett. 94 (2005) 043001.

[15] A. Sorokin, S. Bobashev, K. Tiedtke and M. Richter, Multi-photon ionization of molecular nitrogen by femtosecond soft x-ray FEL pulses, J. Phys. B 39 (2006) 299.

[16] A. Sorokin, M. Wellhöfer, S. Bobashev, K. Tiedtke and M. Richter, X-ray-laser interaction with matter and the role of multiphoton ionization: Free-electron-laser studies on neon and helium, Phys. Rev. A 75 (2007) 051402.

[17] M. Nagasono et al., Resonant two-photon absorption of extreme-ultraviolet free-electron-laser radiation in helium, Phys. Rev. A 75 (2007) 051406.

[18] A. Pietzsch et al., Towards time resolved core level photoelectron spectroscopy with femtosecond $X$-ray free-electron lasers, submitted to New Journal of Physics.

[19] M. Wellhöfer, M. Martins, W. Wurth, A. Sorokin and M. Richter, Performance of the monochromator beamline at FLASH, J. Opt. A: Pure Appl. Opt. 9 (2007) 749.

[20] Z. Silagadze, A new algorithm for automatic photopeak searches, Nucl. Instrum. Meth. A 376 (1996) 451.

[21] M. Morhac, J. Kliman, V. Matousek, M. Veselsky and I. Turzo, Efficient one- and two-dimensional gold deconvolution and its application to $\gamma$-ray spectra decomposition, Nucl. Instrum. Meth. A 401 (1997) 385 . 